\title{
Application of PDE Methods to Visualization of Heart Data
}

\author{
Ognyan Kounchev ${ }^{1}$ and Michael J. Wilson Wilson ${ }^{2}$ \\ ${ }^{1}$ Institue of Mathematics and Informatics - Bulgarian Academy of Sciences, \\ Acad. G. Bonchev 8, 1113 Sofia, Bulgaria, \\ kounchev@math.bas.bg \\ 2 University of Leeds, School of Mathematics, \\ Leeds LS2 9JT, UK, \\ mike@maths.leeds.ac.uk
}

\begin{abstract}
We apply new methods based on Partial Differential Equations techniques (polysplines) to the visualization of the heart surface.
\end{abstract}

\section{A Introduction}

\section{A.1 The Medical Perspective}

There is considerable effort in the area of medical imaging to capture and analyse the motion of the heart, using a variety of imaging techniques, e.g. X-ray Computed Tomography (CT) or Magnetic Image Resonancing (MRI). Because of the complicated motion of the heart and the fact that its surface is deformable, interpreting and analysing image data in order to deduce the underlying motion is not straight-forward and most approaches to the problem are model-based. For example simple shapes such as spheres, ellipsoids, or cylinders are sometimes used to approximate the shape of the Left Ventricle (LV) [2,4,15]. Recently techniques based on the usage of deformable models for reconstructing the 3D surface shape and motion of the $L V$ from $C T$ or $M R I$ data have been developed (e.g. $[1,7,13,17,18,23,20])$. They use a variety of physics-based or geometrical techniques to model the rigid and non-rigid motion of the ventricles (usually the left).

There are two common approaches to modelling the shape of the ventricles. The first aims to construct a generic parametric model to describe the main features of the heart's shape during the cardiac cycle, whilst the second uses MRI scan data to construct a more accurate, and hence more complex, patient-specific model.

The latter approach has been used by Taylor and Yamaguchi [37], Park et al. [21] and Haber et al. [12] amongst others. While these techniques can provide good approximations to the actual geometry in an individual case, a general investigation into the effects of modifications to the shape can be more difficult to perform, due to the large amounts of data typically involved in describing the surface. 
With regard to the former approach, that is to create a generic representation of the heart, the work of Peskin and McQueen [24] is some of the most advanced to date. Their model encompasses both ventricles and atria and also the major arteries connected to the heart. They build up the heart surface by specifying the position of muscle fibres in the heart walls which are connected to the fluid flow using their Immersed Boundary Method. Yoganathan et al. [39] have also adapted Peskin's method to study a thin-walled $L V$ during early systole. The computational time required to perform these calculations, however, makes it difficult to conduct general investigations into different aspects of the motion so other work has used greatly simplified generic ventricle geometry to look at the effects of disease upon the fluid flow in the heart. For example Schoephoerster [28] uses a spherical $L V$ to examine the effects of abnormal wall motion on the flow dynamics.

Other work of note that attempts to combine a geometric model of the heart and its structure with a biomechanical model of its functionality, is that of Ayache and coworkers, who create a volumetric mesh of the ventricles and couple this with electrical and biomechanical models of its functionality, e.g. [29,30,31,3,32]. The aim of the work described here is to create a parametric model of the ventricles of the heart which lies in between the above extremes. Parametric in this sense means that the geometry is defined by a set of 'shape' or 'design' parameters and can be altered by varying these numbers in a controlled way. The work uses a new flexible method for Computer Aided Geometric Design (CAGD) which is based on application of Partial Differential Equations techinques.

Let us stress at this early point of our exposition that the surfaces which we use for representing the heart are interpolating the scanned data and are obtained by a variational principle - by minimization of a curvature functional (to be more precise, the functional is very close to the curvature functional). As such they are in a certain sense surfaces of minimal curvature.

\section{A.2 The Geometric Design Perspective}

In order to better explain the apparatus which we use for modelling the heart geometry, let us make a brief account of its history.

The application of Partial Differential Equations to analyzing and visualizing data has a rather long history. In the late fifties-early sixties, harmonic functions have been used for interpolation of data (for modelling the shape of the cars, aircrafts, etc.) - the basis for using harmonic functions was the existence of analog devices which were able to quickly compute the values of physical quantities which are expressed as harmonic functions ${ }^{3}$, in fact they were much faster than digital computations. However, there was not enough smoothness and flexibility of the harmonic functions as it was clear that only problems of Dirichlet type are in fact soluble in a 'stable way'. Later on with the development of the digital computers there was no need to apply analog devices, and conventional

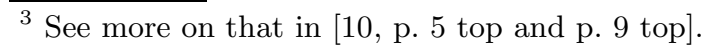


splines (piecewise polynomial) have taken over in popularity as tools for data interpolation and approximation.

A drawback of such methods is that piecewise polynomial splines (and every kind of simpler objects) need, in general, the data to interpolated to be arranged in a special geometry. On the other hand, data from real applications such as Geophysics, often involve huge amounts of data scattered in an unstructured way. For this reason several methods appeared over the years for handling such data. Some of them are closely related to Partial Differential Equations. One of the first, and nowadays very popular method, is the so-called Minimum curvature method, see Briggs [6], which is a solution of a minimization problem. Namely, suppose that we are given some measurements $c_{j}$ at data points $\left(x_{j}, y_{j}\right)$ for $j=1,2, \ldots, N$, in a plane domain $D$, see Figure 1 .

Then the problem of finding the surface of minimum curvature may be formulated as follows: Find a surface $f(x, y)$ which satisfies

$$
f\left(x_{j}, y_{j}\right)=c_{j} \quad \text { for } j=1,2, \ldots, N,
$$

and which has minimum curvature in the differential-geometric sense of the word. Since the exact expression for the curvature is rather complicated, one takes the expression $\Delta f(x, y)$ to be a relatively good approximation to it. Or written more precisely, we have the following extremal problem,

$$
\left\{\begin{array}{l}
\inf _{f} \int_{D}[\Delta f(x, y)]^{2} d x d y \\
f\left(x_{j}, y_{j}\right)=c_{j} \quad \text { for } j=1,2, \ldots, N \\
\text { and some boundary condition for } f \text { on } \partial D .
\end{array}\right.
$$

There are very efficient algorithms for solving such problems and the results obtained are rather satisfactory for the purposes of Geophysics; see [6] as an initial reference, and for more recent account of these methods see [11], [34]. Let us mention that another method called "Kriging" has appeared about the same time, see the package "Surfer" for a software implementation of these and other algorithms, [35].

In the mid-seventies, the ultimate generalization of the above approach was obtained in the works of Duchon [8] and Meinguet [19]. They laid the mathematical foundation of a new area called nowadays Radial Basis Functions. This direction has developed theoretically as well as practically, see the survey papers [25] and [9]. Let us note that the above methods have some drawbacks in analyzing huge amounts of data which have points of concentration (in the mathematical sense of this word), in particular if the data are densely located on some curves. Their computation is based on solving large linear systems, and with increasing the number of data points the condition number becomes very small. Their major drawback for the purposes of CAGD is that the surfaces which they create show some artificial oscillations (called "pockmarks" by the geophysicists) occurring mainly near the data points.

Parallel to the above development, during the last decade new interest has appeared towards methods based on Partial Differential Equations in Approximation Theory, Spline Theory and especially in CAGD, see [5]. In these new 


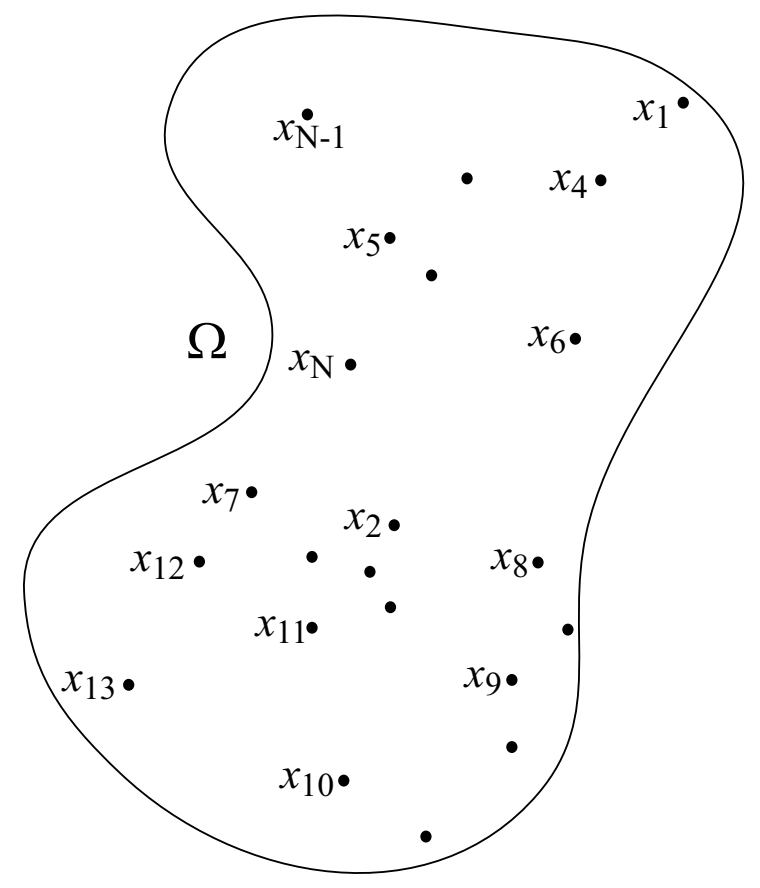

Fig. 1. Scattered data in a plane domain.

methods solutions of PDEs are used for interpolation and approximation of the measured data. As an alternative to the "classical" spline theory which relies upon polynomials a new method has appeared which relies upon solutions of PDEs, see [16]. It is based on the minimum curvature functional but has a different concept of the data. The polysplines are a solution of a problem similar to the one in (1) but the data (when we consider the two-dimensional case) are lying on a set of curves, see e.g. the geometrical configuration on Figure 2.

We will assume throughout the present paper that the exterior-most curve $\Gamma_{N}$ is the boundary of the domain $D$, i.e. $\partial D=\Gamma_{N}$. Let us note that the domain $D$ may be unbounded and the curve $\Gamma_{N}$ may consist of several disconnected pieces. In the above setting, the polysplines are a solution to the following problem

$$
\left\{\begin{array}{c}
\inf _{f} \int_{D}[\Delta f(x, y)]^{2} d x d y \\
f(x, y)=g_{j} \text { on } \Gamma_{j} \text { for } j=1,2, \ldots, N, \\
\text { and some boundary condition for } f \text { on } \partial D .
\end{array}\right.
$$

Here the "data functions" $g_{j}$ are prescribed on the curves $\Gamma_{j}$.

The theory of polysplines has been extensively studied in the recent monograph [16]. The polysplines have proved to be efficient for smoothing data in Magnetism, in Geophysics, as well as for application to a number of CAGD data, in 


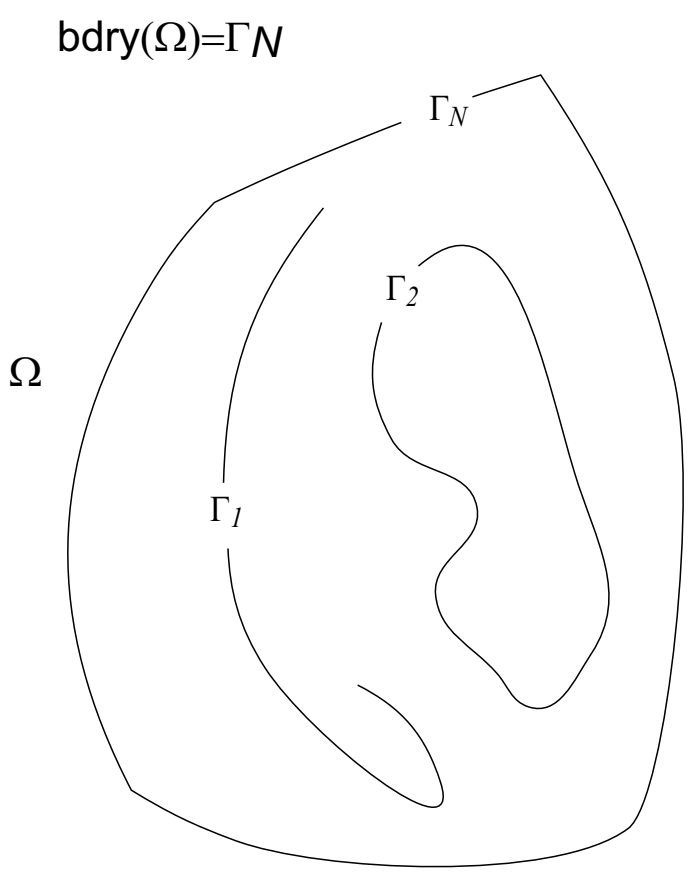

Fig. 2. The data are lying on the curves $\Gamma_{j}$. They are considered to be "scattered".

particular in cases where the data curves have rather irregular form, see Chapter 6 of $[16]$.

The present paper is devoted to the application of the polysplines to heart data. These data are measured by scanning the surface of the heart at different levels, i.e. for different $z$ in the three dimensional coordinates $(x, y, z)$. In the data available we have measurements at 7 different levels, i.e. for $z=Z^{1}, Z^{2}, \ldots, Z^{7}$. The main point is to extend these scanned data and to create a visualization of the heart (the left ventricle) as well as to observe the dynamics of the heart activity by creating its form at different phases. The data used in this paper has been extracted from MRI images of horizontal sections through a human heart taken at various stages in the cardiac cycle. ${ }^{4}$

The method which we introduce below is applicable to more general situations when the data measured need not to be at the same level in the $z$ direction.

\footnotetext{
${ }^{4}$ The data used supplied by the Department of Medical Physics at Leeds General Infirmary.
} 


\section{B Introduction of Appropriate Coordinates for the Heart Surface}

In the present Section we discuss the introduction of appropriate coordinates in which the surface of the heart is conveniently represented ${ }^{5}$. We will see that in these new coordinates the part of the heart surface of interest $(L V)$ becomes a function on a cylinder. The scanned data will be respectively the sections at some levels of this surface. Thus we will be able to apply to this "heart surface function" the ready-made device called "polysplines on cylinder". ${ }^{6}$

We assume that all sectional curves $G_{j}$ (which are closed curves) are made in the $z$ direction at the level $z=Z^{j}$ for $j=1,2, \ldots N$, where

$$
Z^{1}<Z^{2}<\ldots<Z^{N}
$$

In all data available we have in particular

$$
\begin{aligned}
& Z^{1}=0.00, \quad Z^{2}=0.01, \quad Z^{3}=0.02, \\
& Z^{4}=0.03, \quad Z^{5}=0.04, \quad Z^{6}=0.05, \\
& Z^{7}=0.07 \text {. }
\end{aligned}
$$

On the next Figure 3 we have a picture showing the projections of the 7 curves on the $(x, y)$ plane for Phase 2 of the heart dynamics (needless to say these curves and their projections vary with the phases).

Further we make the assumption that all curves $G_{j}$ are star-shaped, i.e. for every $G_{j}$ there exists a point $\left(X^{j}, Y^{j}, Z^{j}\right)$ such that for every point $P \in G_{j}$ the line interval connecting $P$ and $\left(X^{j}, Y^{j}, Z^{j}\right)$ lies entirely inside the curve $G_{j}$.

Remark 1. All practical data which we use further in our study satisfy this assumption - the curves $G_{j}$ are star-shaped. Even more, we may observe that they are star-shaped with respect to their center of gravity which provides an algorithm for finding the center of star-shaped-ness.

After we have done such simplifying assumption our algorithm runs as follows: We choose the new coordinate system by first finding the points $\left(X^{j}, Y^{j}, Z^{j}\right)$ for all sections $G_{j}$. Then we join them in the space by an interpolation spline in the $z$ direction, for example, by interpolation cubic spline. In fact we have to take two such spline functions of the variable $z$, namely $g_{1}(z)$ and $g_{2}(z)$. They will give the curve $\left(g_{1}(z), g_{2}(z), z\right)$ described by the parameter $z$. The interpolation condition means

$$
g_{1}\left(Z^{j}\right)=X^{j}, \quad g_{2}\left(Z^{j}\right)=Y^{j} \quad \text { for } j=1,2, \ldots, N .
$$

\footnotetext{
${ }^{5}$ Speaking mathematically precisely, we define a diffeomorphism on the heart surface which maps it to a cylinder surface. We provide a description of such diffeomorphism in a case somewhat more general than the concrete data require.

${ }^{6}$ In [16] see Sections 5.2 and 5.3, p. 60, for a detailed definition of "polysplines on cylinder"; see Section 22.2, p. 448 for the proof of the existence of "interpolation polysplines on cylinder".
} 


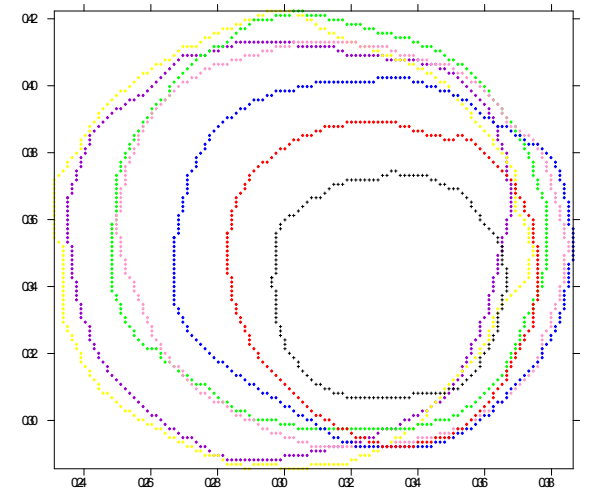

Fig. 3. This is the projection of the section curves $G_{j}$ on the $(x, y)$ plane for the Phase 2 of the heart dynamics.

Clearly, the simplest situation is when we are able to choose all centers $\left(X^{j}, Y^{j}, Z^{j}\right)$ coinciding, i.e.

$$
\left(X^{j}, Y^{j}, Z^{j}\right)=\left(X^{0}, Y^{0}, Z^{j}\right) \quad \text { for all } j=1,2, \ldots, N .
$$

In such a case we have a trivial step in the algorithm since the "joining spline" is a constant, namely

$$
g_{1}(z)=X^{0}, \quad g_{2}(z)=Y^{0} .
$$

For example, for our particular data we may choose

$$
X^{0}=0.33, \quad Y^{0}=0.34
$$

these coordinates are obviously located on Figure 3.

Then we will introduce new variables by using cylindric coordinates. At every level $z$ these new parameters have the form

$$
\begin{gathered}
\theta=\arctan \frac{y-Y^{j}}{x-X^{j}} \quad \text { for }\left(x, y, Z^{j}\right) \in G_{j} \\
r_{j}=r_{j}(\theta)=r\left(Z^{j}, \theta\right)=\sqrt{\left(x-X^{j}\right)^{2}+\left(y-Y^{j}\right)^{2}} .
\end{gathered}
$$

In the above notations we have assumed implicitly that the part of the heart surface of interest $(L V)$ is described by the function $r(z, \theta)$ in the cylindric coordinates $(z, \theta, r)$. Thus the given data are in the form of $N$ periodic functions 
$r_{j}(\theta)$ for $j=1,2, \ldots, N$ and $0 \leq \theta \leq 2 \pi$, corresponding to the curves $G_{j}$. Finally, we obtain the image of the curves $G_{j}$ (which we will denote by $\widetilde{G_{j}}$ ) in the new coordinates $(z, \theta, r)$, namely, for every $j$ the curve $G_{j}$ from the heart surface is mapped into the graph of the function $r_{j}$, i.e. into the curve

$$
\widetilde{G_{j}}:=\left\{\left(Z^{j}, \theta, r_{j}(\theta)\right): 0 \leq \theta \leq 2 \pi\right\} .
$$

See Figure 4 where we have the images of $\widetilde{G_{j}}$ on the cylinder projected on the plane $(z, \theta)$ - these are $N=7$ parallel lines.

With this we finish the introduction of the new coordinates.

The visualization in the new coordinates $(z, \theta, r)$ is relatively simple, and to that end we are able to apply directly the polysplines. The result will be a periodic polyspline, namely, a function $r(z, \theta)$ defined for

$$
\begin{aligned}
Z^{1} & \leq z \leq Z^{N}, \\
0 & \leq \theta \leq 2 \pi,
\end{aligned}
$$

which is $2 \pi$-periodic with respect to the variable $\theta$. Recall that in our particular data we have $N=7$ with $Z^{1}=0$ and $Z^{7}=0.07$.

Now we need the inverse map in order to come back to the original variables. By using the original variables $(x, y, z)$ we obtain the following representation of the heart surface in the variables $(z, \theta, r)$,

$$
\begin{aligned}
Z^{1} & \leq z \leq Z^{N}, \quad 0 \leq \theta \leq 2 \pi \\
\theta & =\arctan \frac{y-g_{2}(z)}{x-g_{1}(z)} \\
r(z, \theta) & =\sqrt{\left(x-g_{1}(z)\right)^{2}+\left(y-g_{2}(z)\right)^{2}} .
\end{aligned}
$$

Solving with respect to $x$ and $y$ we obtain the surface

$$
H=\left\{\begin{array}{l}
x=g_{1}(z)+r(z, \theta) \cos \theta \\
y=g_{2}(z)+r(z, \theta) \sin \theta \\
Z^{1} \leq z \leq Z^{N}, \quad 0 \leq \theta \leq 2 \pi .
\end{array}\right.
$$

\section{Polysplines on a Cylinder}

Let us say some words about the polysplines on a cylinder.

As we saw above the part of the heart surface of interest $(L V)$ is mapped to a periodic function on the rectangle $[0,0.07] \times[0,2 \pi]$ with coordinates $(z, \theta)$. The measured data lie on the lines $\gamma_{j}$ defined by $z=Z^{j}$, so we have

$$
\gamma_{j}:=\left\{(z, \theta): z=Z^{j}, 0 \leq \theta \leq 2 \pi\right\}
$$

or shortly

$$
\gamma_{j}=\left\{z=Z^{j}\right\}
$$




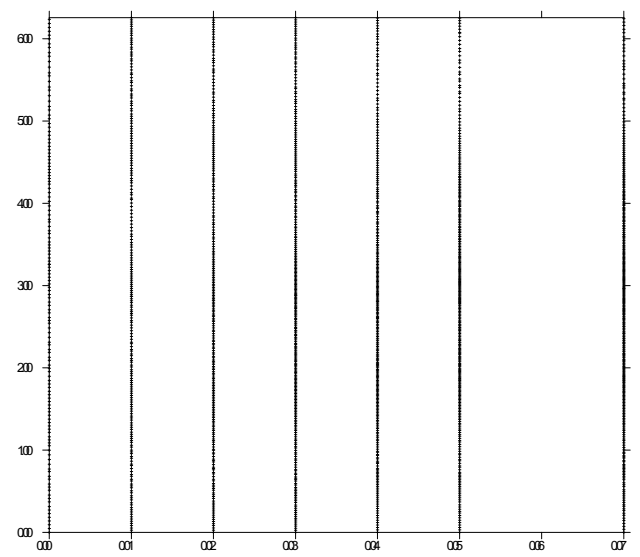

Fig. 4. The projections of the seven curves $\widetilde{G_{j}}$ are shown in the plane $(z, \theta)$.

Recall that $\gamma_{j}$ is the projection of the curve $\widetilde{G_{j}}$. We have

$$
\begin{aligned}
& \gamma_{1}=\{z=0\}, \quad \gamma_{2}=\{z=0.01\}, \quad \gamma_{3}=\{z=0.02\} \\
& \gamma_{4}=\{z=0.03\}, \quad \gamma_{5}=\{z=0.04\}, \quad \gamma_{6}=\{z=0.05\}, \quad \gamma_{7}=\{z=0.07\}
\end{aligned}
$$

To that configuration we apply "polysplines on a cylinder", as we already refered to [16, Sections 5.2 and 5.3 , p. 60]. To give a short summary of the main result: the polyspline in our case will be a function $u(z, \theta)$ consisting of 6 pieces $u_{j}(z, \theta)$ where $u_{j}(z, \theta)$ is a function defined between the lines $\gamma_{j}$ and $\gamma_{j+1}$ for $j=1,2, \ldots, 6$; and $u_{j}$ satisfies there

- the biharmonic equation

$$
\Delta^{2} u_{j}(z, \theta)=\left(\frac{\partial^{2}}{\partial z^{2}}+\frac{\partial^{2}}{\partial \theta^{2}}\right)^{2} u_{j}(z, \theta)=0 \quad \text { between } \gamma_{j} \text { and } \gamma_{j+1}
$$

- the interpolation to the experimental data

$$
u_{j}\left(Z^{j+1}, \theta\right)=u_{j+1}\left(Z^{j+1}, \theta\right)=r_{j}\left(Z^{j+1}, \theta\right) \quad \text { for all } 0 \leq \theta \leq 2 \pi \text {, }
$$

- and the smoothness condition

$$
u \in C^{2}
$$


i.e.

$$
\begin{aligned}
u_{j}\left(Z^{j+1}, \theta\right) & =u_{j+1}\left(Z^{j+1}, \theta\right) & \text { for all } 0 \leq \theta \leq 2 \pi, \\
\frac{\partial}{\partial z} u_{j}\left(Z^{j+1}, \theta\right) & =\frac{\partial}{\partial z} u_{j+1}\left(Z^{j+1}, \theta\right) & \text { for all } 0 \leq \theta \leq 2 \pi, \\
\frac{\partial^{2}}{\partial z^{2}} u_{j}\left(Z^{j+1}, \theta\right) & =\frac{\partial^{2}}{\partial z^{2}} u_{j+1}\left(Z^{j+1}, \theta\right) & \text { for all } 0 \leq \theta \leq 2 \pi,
\end{aligned}
$$

The main result of [16] is the existence of interpolation polyspline: If we are given the scanned data $r\left(Z^{j}, \theta\right)$ then we may find the polyspline with the above properties. Let us remark that the functions $u_{j}$ are real analytic, i.e. the polysplines enjoy infinite smoothness away from the "interfaces" $\gamma_{j}$ and on $\gamma_{j}$ they are $C^{2}$.

\section{The Results}

In the $(z, \theta, r)$ coordinates the result of smoothing of the given data $r\left(Z^{j}, \theta\right)$ with the Kriging method as implemented in [35] is shown on Figure 5. What we have on this Figure is in fact an approximation to Phase 1 of the heart surface in the $(z, \theta, r)$ coordinates.

The result of smoothing the same data with interpolation polysplines is shown on Figure 6.

One has to compare the two Figures 5 and 6 and to see that the surface obtained by the polyspline method is visually smoother than the one obtained with the Kriging method.

Next we provide the visualization result using the original $(x, y, z)$ coordinates by means of formula (4). We provide the results in a series of phases of the heart dynamics viewed from different perspectives.

Let us note that we take the same "center of star-shaped-ness" $\left(X^{0}, Y^{0}\right)$ in all cases, namely

$$
X^{0}=0.33, \quad Y^{0}=0.34 .
$$

\section{D.1 Visualization of the Left Ventrical at Different Phases}

On Figure 7 Phases 1,3,5,7 are visualized.

On Figure 8 we have Phases 9,11,13 visualized.

Conclusion 1 We have shown that by means of appropriate coordinate change the Left Ventricle may be conveniently represented in cylindrical coordinates where the axis variable is $z$. In this setting if scanning has been performed at different levels in $z$ then we have seen that a ready-made tool called "polyspline on a cylinder" is available. This polyspline is a surface which interpolates the measured data and provides as seen from Figures 7 and 8 visually very satisfactory approximation to the heart surface. The above scheme is applicable to a lot more general setting for modelling surfaces which may be transformed by a diffeomorphism to become "functions on a cylinder". 
Acknowledgement 2 The current research of the first author has been sponsored by the Nuffield foundation during his stay at the University of Leeds, and by the Alexander von Humboldt Foundation during his stay at the University of Duisburg. The authors would like to acknowledge the help of Chris Evans (Applied Mathematics, University of Leeds), A. Radjenovic, and J.P. Ridgeway of the Department of Medical Physics of the University of Leeds, in providing the data for these studies.

\section{References}

1. Amini, A., Duncan, J., "Pointwise tracking of Left-Ventricular Motion in 3D", Proc. IEEE Workshop on Visual Motion, Princeton, NJ, 1991, pp. 294-298.

2. Arts, T., Hunter, W. C., Douglas, A., Muijtjens, M. M., Reneman, R.S., "Description of the deformation of the left ventricle by a kinematic model", J. Biomechanics, Vol. 25, No. 10, 1992, pp. 1119-1127.

3. N. Ayache, D. Chapelle, F. Clément, Y. Coudière, H. Delingette, J.A. Désidéri, M. Sermesant, M. Sorine, and J. Urquiza, "Towards Model-Based Estimation of the Cardiac Electro-Mechanical Activity from ECG Signals and Ultrasound Images", In T. Katila, I.E. Magnin, P. Clarysse, J. Montagnat, and Nenonen J., editors, Functional Imaging and Modeling of the Heart (FIMH'01), Helsinki, Finland, VOl 2230 Lecture Notes in Computer Science, pp. 120-127, 2001. Springer.

4. Beyar, R., Sideman, S., "Effect of the twisting motion on the non-uniformities of transmural fiber mechanics and energy demands-A theoretical Study", IEEE Trans. Biomed. Eng., Vol, 32, 1985, pp. 764-769.

5. Bloor, M.I.G.; Wilson, M.J. "The PDE method in geometric and functional design". Goodman, Tim (ed.) et al., The mathematics of surfaces. VII. Proceedings of the 7th conference, Dundee, Great Britain, September 1996. Winchester: Information Geometers, Limited. 281-307 (1997).

6. Briggs, J.C. "Machine contouring using minimum curvature", Geophysics, 39 (1974), 39-48.

7. Cohen, L.D. and Cohen, I., "A Finite Element Method Applied to New Active Contour Models and 3d Reconstruction from Cross-sections", Proc. 3rd Int. Conf. on Computer Vision, Osaka, Japan, 1990, pp. 587-591.

8. Duchon, J. "Interpolation des fonctions de deux variables suivant le principe de la flexion des plaques minces", R.A.I.R.O. Analyse numerique, vol. 10, no. 12 (1976), pp. $5-12$.

9. Dyn, N. "Interpolation and approximation by radial and related functions", In: Approximation Theory VI, C. K. Chui, L. L. Schumaker and J. D. Ward (eds.), Academic Press, New York, 1989, pp. 211-234.

10. Farin, G. E. "Curves and Surfaces for Computer Aided Geometric Design: a Practical Guide". 4th ed, Boston, MA: Academic Press, 1997.

11. Gonzalez-Casanova, P.; R. Alvarez, "Splines in geophysics", Geophysics, 50, No. 12 (1985), 2831-2848.

12. Haber E., Metaxas D.N. and Axel L., "Motion Analysis of the Right Ventricle from MRI Images", Lecture Notes in Computer Science, 1998, Vol. 1496, pp 177-188.

13. Huang, W.C. and Goldgof, D., "Adaptive-Size Meshes for Rigid and Nonrigid Shape Analysis and Synthesis", IEEE Transactions on Pattern Analysis, Vol. 15, 1993, pp. 611-616. 
14. Kim W.Y et al., "Left-ventricular blood-flow patterns in normal subjects", Journal of the American College of Cardiology, 1995, Vol. 26, 1, pp 224-37.

15. Kim, H. C., Min, B. G., Lee, M. M., Seo, J. D., Lee, Y. W., Han, M. C., "Estimation of local cardiac wall deformation and regional stress from biplane coronary cineangiograms", IEEE Trans. Biomed. Eng., Vol. 32, 1985, pp. 503-511.

16. Kounchev, O. "Multivariate Polysplines. Applications to Numerical and Wavelet Analysis", Academic Press, San Diego-London, 2001.

17. McInerney, T. and Terzopoulos, D., "A Finite Element Model for 3D Shape Reconstruction and Nonrigid Motion Tracking", Proc. 4th Internation Conference on Computer Vision, Berlin, Germany, 1993, pp. 518-523.

18. McInerney, T. and Terzopoulos, D. "A Dynamic Finite-Element Surface Model for segmentation and tracking in Multi-dimensional Medical Images with application to Cardiac 4D Image-Analysis", Computerized Medical Imaging and Graphics, Vol 19, 1995, pp. 69-83.

19. Meinguet, J. "Multivariate interpolation at arbitrary points made simple", Z. Angew. Math. Phys. 30 (1979), pp. 292-304.

20. Park, J., Metaxas, D., Young, A. A., Axel, L., "Deformable models with parameter functions for cardiac motion analysis from tagged MRI data", IEEE Trans. Medical Imaging, Vol. 15, 1996, pp. 278-289.

21. Park J., Metaxas D., Young A.A and Axel L, "Deformable Models with Parameter Functions for Cardiac Motion Analysis from Tagged MRI Data", IEEE Transactions on Medical Imaging, 1996, Vol. 15, 3.

22. Pedley T.J., "The Fluid Dynamics of Large Blood Vessels", Cambridge University Press, 1980.

23. Pentland, A., Horowitz, B., Sclaroff, S., "Recovery of Nonrigid Motion and Structure from Contour", Proc. IEEE Workshop on Visual Motion, Princeton, NJ, 1991, pp. 288-293.

24. Peskin C.S. and McQueen D.M., "Cardiac Fluid Dynamics", Critical Reviews in Biomedical Engineering, 1992, Vol. 20, 5-6, pp 451 et seq.

25. Powell, M. J. D. "The theory of radial basis function approximation in 1990". In: Advances in numerical analysis. Vol. 2: Wavelets, subdivision algorithms, and radial basis functions, Proc. 4th Summer Sch., Lancaster/UK 1990, pp. 105-210 (1992).

26. Roma A.M, Peskin C.S. and Berger M.J., "An Adaptive Version of the Immersed Boundary Method", Journal of Computational Physics, 1999, Vol. 147, 2, pp 509534.

27. J. Saghri and J. Freeman, "Analysis of the Precision of Generalized Chain Codes for the Representation of Planar Curves", IEEE Transactions on Pattern Analysis and Machine Intelligence, 1981, PAMI-3, pp. 533-539

28. Schoephoerster R.T., Silva C.L. and Ray G., "Evaluation of Left-Ventricular Function based on Simulated Systolic Flow Dynamics Computed from Regional WallMotion", Journal of Biomechanics, 1994, Vol. 27, 2, pp 125-136.

29. M. Sermesant, Y. Coudière, H. Delingette, N. Ayache, J. Sainte-Marie, D. Chapelle, F. Clément, and M. Sorine, "Progress Towards Model-Based Estimation of the Cardiac Electromechanical Activity from ECG Signals and 4D Images", In Marc Thiriet, editor, Modelling and Simulation for Computer-aided Medicine and Surgery (MS4CMS'02), Vol 12 of ESAIM: PROC, pp. 153-162, 2002. European Series in Applied and Industrial Mathematics

30. M. Sermesant, Y. Coudière, H. Delingette, and N. Ayache, "Progress towards an Electro-Mechanical Model of the Heart for Cardiac Image Analysis", In IEEE International Symposium on Biomedical Imaging (ISBI'02), pp. 10-14, 2002. 
31. M. Sermesant, C. Forest, X. Pennec, H. Delingette, and N. Ayache, "Biomechanical Model Construction from Different Modalities: Application to Cardiac Images", In Takeyoshi Dohi and Ron Kikinis, editors, Medical Image Computing and Computer-Assisted Intervention (MICCAI'02), Vol 2488 Lecture Notes in Computer Science, Tokyo, pp. 714-721, September 2002. Springer.

32. M. Sermesant, Y. Coudière, H. Delingette, N. Ayache, and J.A. Désidéri, "An Electro-Mechanical Model of the Heart for Cardiac Image Analysis". In W.J. Niessen and M.A. Viergever, editors, 4th Int. Conf. on Medical Image Computing and Computer-Assisted Intervention (MICCAI'01), Vol 2208 of Lecture Notes in Computer Science, Utrecht, The Netherlands, pp. 224-231, October 2001.

33. Smith J.J. and Kampine J.P., "Circulatory Physiology : the essentials", Baltimore : Williams and Wilkins, 1990, 3rd ed.

34. Smith, M. H. F.; P. Wessel, "Gridding with continuous curvature splines in tension", Geophysics, 55 (1990), No. 3, 293-305.

35. "SURFER - Golden Software", Golden, Colorado, Release of 1997.

36. Taylor D.E.M. and Wade J.D., "The pattern of flow around the atrioventricular valves during diastolic ventricular filling", Journal of Physiology, 1970, Vol. 207, pp 71-2.

37. Taylor T.W. and Yamaguchi T., "Flow Patterns in 3-dimensional Left-ventricular Systolic and Diastolic flows determined from Computational Fluid-Dynamics", Biorheology, 1995, Vol. 32, 1, pp 61-71.

38. Vierendeels J.A., Riemslagh K., Dick E. and Verdonck P.R. "Computer Simulation of Intraventricular Flow and Pressure Gradients During Diastole", Journal of Biomechanical Engineering-Transactions of the ASME, 2001, Vol. 122, 6, pp 667-674.

39. Yoganathan A.P., Lemmon J.D., Kim Y.H., Walker P.G., Levine R.A., Vesier C.C, "A Computational Study of a Thin-Walled 3-Dimensional Left Ventricle during Early Systole", Journal of Biomechanical Engineering-Transactions of the ASME, 1994, Vol. 116, 3, pp 307-314. 


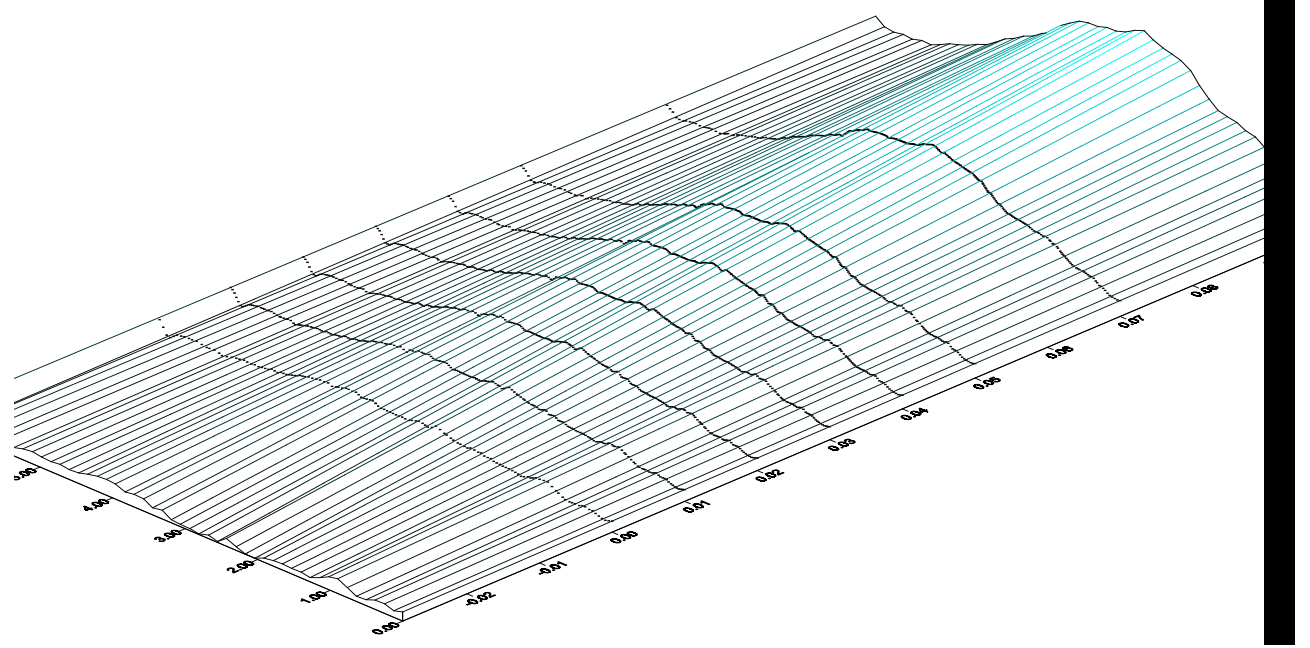

Fig. 5. This is the smoothing with Kriging in the $(z, \theta, r)$ coordinates. The seven curves $\widetilde{G_{j}}$ with the measured data are displayed on the surface.

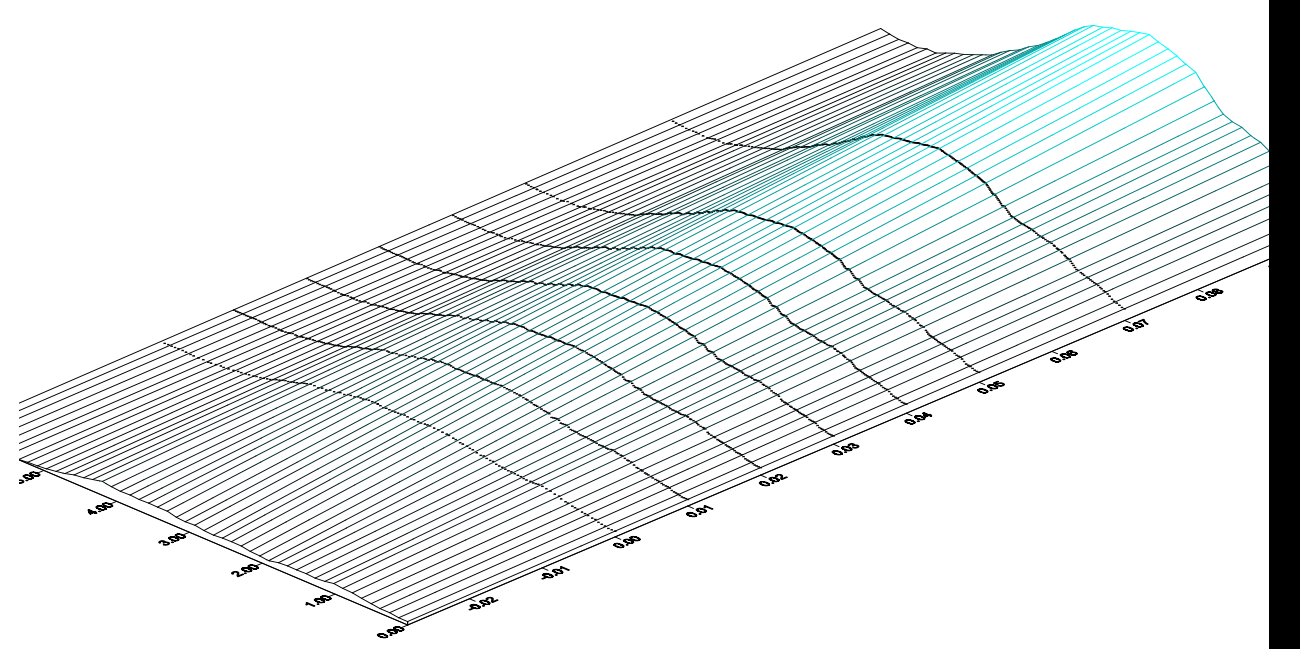

Fig. 6. This is the smoothing with the polyspline in the $(z, \theta, r)$ coordinates. The seven curves $\widetilde{G_{j}}$ with the measured data are displayed on the surface. 


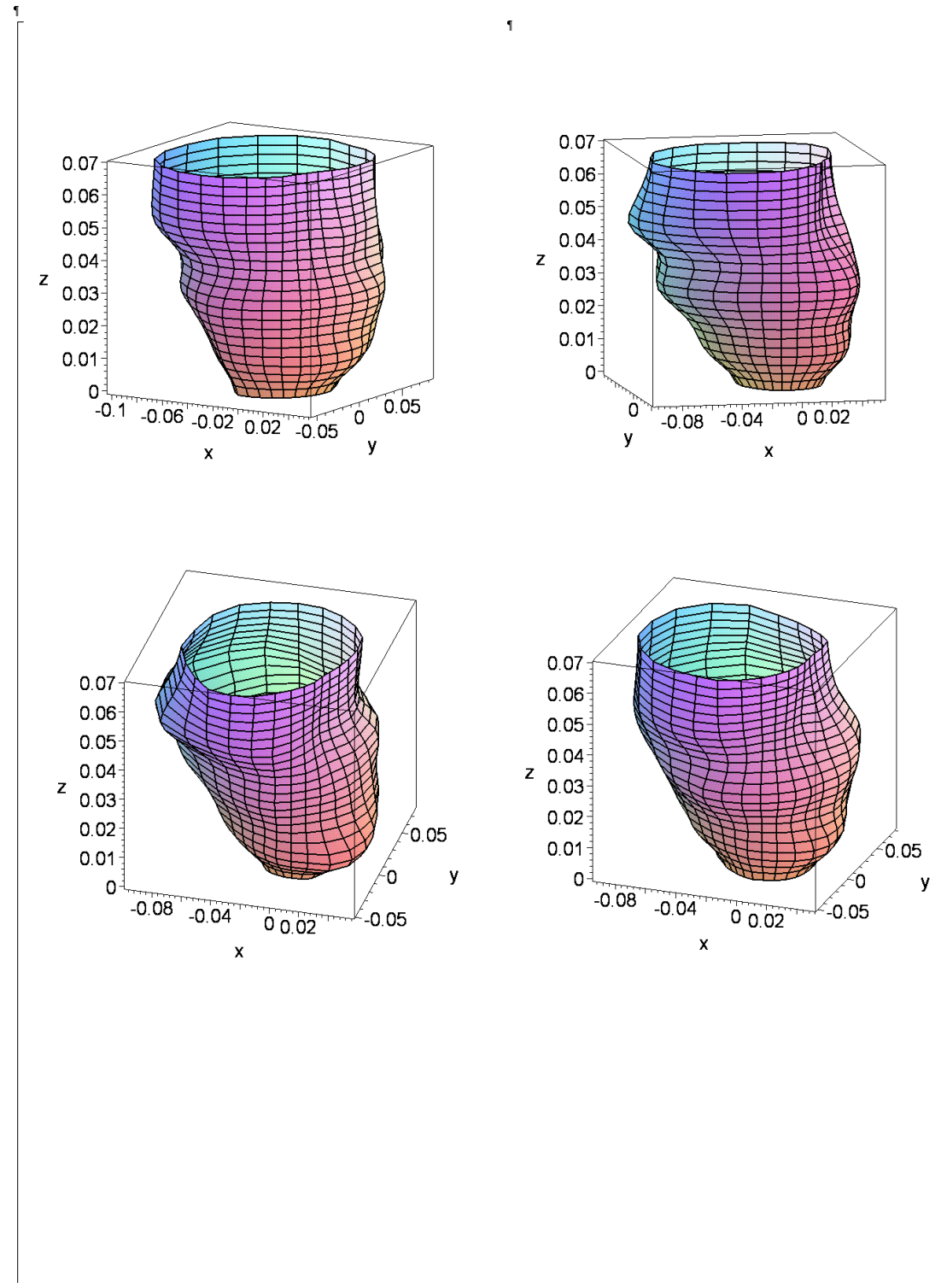

Fig. 7. This is the heart surface of Phases 1,3,5,7 resulting from the application of the polysplines. 


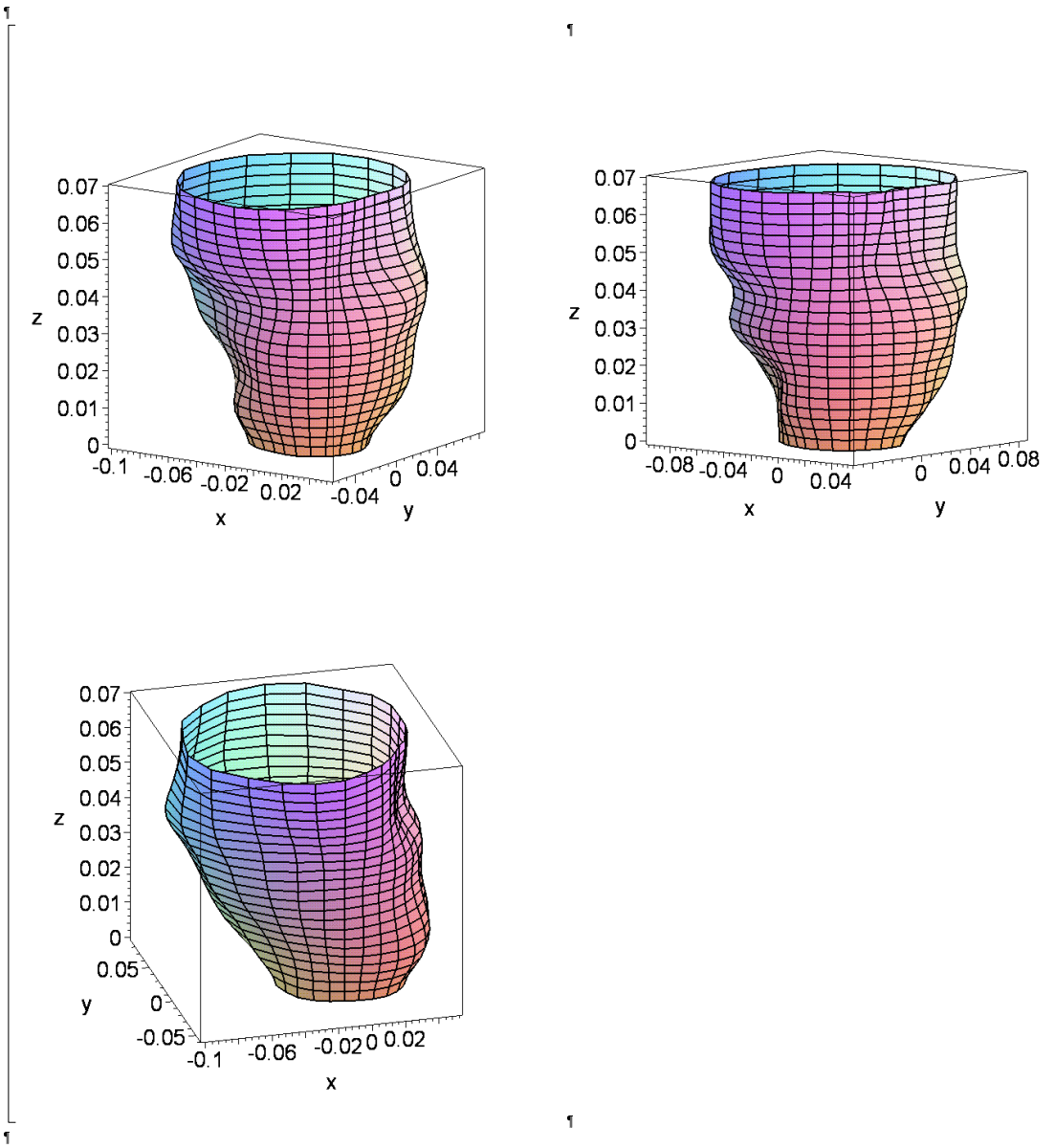

Fig. 8. This is the heart surface of Phases 9,11,13 resulting from the application of the polysplines. 